Check for updates

Cite this: RSC Adv., 2019, 9, 38677

Received 7th October 2019

Accepted 14th November 2019

DOI: 10.1039/c9ra08123b

rsc.li/rsc-advances

\section{Experimental and DFT studies of carbon nanotubes covalently functionalized with an imidazole derivative for electrochemical stability and green corrosion inhibition as a barrier layer on the nickel alloy surface in a sulphuric acidic medium}

\begin{abstract}
Palaniappan N., (D) ${ }^{* a}$ I. S. Cole, (DD ${ }^{* b}$ A. E. Kuznetsov (DD ${ }^{c}$ and K. R. Justin Thomas (D) ${ }^{d}$
Nickel-based alloys are used for various applications such as in automobiles, superalloys and ship making. Their stability in acidic environments, however, is often not sufficient due to their native oxide films not withstanding harsh conditions. Carbon-based materials, in contrast, have shown high stability in acidic environments. In the present work, 4,5-diphenyl-imidazole-functionalized carbon nanotubes were investigated as a corrosion-inhibiting barrier layer on nickel alloy surfaces. We studied the corrosion inhibition efficiency of the imidazole-functionalized carbon nanotubes coated on nickel alloys. The materials were characterized using microscopy and spectroscopy methods. DFT studies were performed as well. The corrosion inhibition was evaluated using electrochemical methods. The imidazolefunctionalized CNTs were shown to be crystalline and to have tubular structures. They displayed excellent corrosion inhibition on the nickel alloy surface.
\end{abstract}

\section{Introduction}

The past decades have witnessed continuous problems with corrosion in harsh environments due to the generally poor quality of available inhibitors of corrosion and due to the instability of coating materials. Nickel (Ni) alloys have been shown to display strong resistance to corrosion in the open-air environment but weak such resistance in acidic conditions due to the increased rate of penetration of corrosive ions. The hightemperature corrosion resistance of the cobalt (Co)-decorated nickel-aluminum (Ni-Al)-coated $\mathrm{Ni}$ alloy was studied by Zhou et $a l .{ }^{1}$ Furthermore, the ability of yttrium (Y) to slow down the high-temperature $\left(750^{\circ} \mathrm{C}\right)$ corrosion of the nickel-iron $(\mathrm{Ni}-\mathrm{Fe})$ alloy was studied by $\mathrm{Xu}$ et al. The authors stated that upon the addition of $\mathrm{Y}$, the corrosion inhibition increased, operating using a reduced temperature scale. ${ }^{2}$ They suggested as well the occurrence of an increase in the corrosion inhibition efficiency with an increased weight percentage of the composite materials. Then, corrosion inhibition in a salt medium of the zinc-

${ }^{a}$ School of Chemical Sciences, Central University of Gujarat, India. E-mail: palaniappancecri@rediffmail.com

${ }^{b}$ Advance Manufacturing and Fabrication Research and Innovation, RMIT University, Melbourne, Victoria 3100, Australia. E-mail: ivan.cole@rmit.edu.au

'Department of Chemistry, Universidad Técnica Federico Santa Maria, Campus Vitacura, Santiago, Chile

${ }^{d}$ Organic Materials Laboratory, Department of Chemistry, Indian Institute of Technology Roorkee, Roorkee 247667, India nickel (Zn-Ni) alloy coated with Zn-citrate was investigated by Khan et al. $^{3}$ Furthermore, friction studies of the 5,50-dimethylhydantoin (DMH)-coated $\mathrm{Zn}-\mathrm{Ni}$ alloy were carried out by $\mathrm{An}$ et $a l^{4}{ }^{4}$ They suggested the occurrence of an increase as well in friction efficiency with an increased concentration of additive. With a chromium-nickel ( $\mathrm{Cr}-\mathrm{Ni}$ ) alloy protected by liquid polymer, corrosion of the pure $\mathrm{Ni}$ alloy at $700{ }^{\circ} \mathrm{C}$ resulting from the addition of sodium sulphate was investigated by YuasacaIchikawa et $a .^{5-7}$ The results indicated that the effect of sodium sulphate decreased as phase transfer occurred at high temperature. Furthermore, inhibition of Ni-Co alloy corrosion in lithium (Li)-based molten salt was investigated by Lee et al. Their results suggested that high concentrations of $\mathrm{Cr}$ increased granular corrosion. ${ }^{8}$ Liu concluded that for the Ni alloy, the surface pretreatment using a ball mill was the factor increasing the corrosion resistance, and a further machine pressing treatment at high pressure increased the corrosion resistance. ${ }^{9}$ The pretreated surface acted as a nanolayer. Recently, carbonfunctionalized materials have shown increasing use in many fields due to their high surface areas and long life spans. Graphene oxide, carbon nanotubes (CNTs), and graphitic carbon have been studied for corrosion inhibition properties in different media. Carbon nanotubes have been shown to possess a unique property: they can be functionalized with nanoparticles and heterocyclic organic compounds. ${ }^{\mathbf{1 0 - 1 3}}$ Several investigators have conducted corrosion inhibition studies of nanoparticle-decorated CNTs and polyaniline-functionalized graphene oxide due their being highly conductive materials. ${ }^{\mathbf{1 4}}$ 
Liu et al., for example, investigated the corrosion inhibition of a graphene-oxide-coated aluminium alloy in a salt environment. ${ }^{15}$ The results suggested a corrosion inhibition efficiency here of about $96 \%$, attributed to the high surface areas of the graphene oxide materials. Furthermore, reduced graphene oxide functionalized with polyvinyl alcohol was investigated as a corrosion barrier layer on aircraft grade aluminium alloy in $2 \mathrm{M} \mathrm{NaCl}$ environments. ${ }^{16}$ The results suggested the occurrence of an increase in inhibition efficiency with an increase in the weight percentage of this inhibitor. Zhou et al. concluded that CNTs added to $\mathrm{Mg}$ grain boundaries improved the corrosion resistance by forming complexes on the $\mathrm{Mg}$ grain boundaries. ${ }^{17}$ Meng et al. studied the corrosion inhibition efficiency of cupric sulphate coated on nickel alloy; a high concentration of cupric sulphate here showed a 55\% inhibition efficiency in a solution of $3.5 \%$ NaCl. ${ }^{17 b}$ Carbon materials have been extensively used in various biological applications. They can also be used as ecofriendly materials. In the present study, 4,5diphenylimidazole-functionalized CNTs were investigated as a coating material for nickel alloys. This material can be expected to serve as an eco-friendly coating material in the future, and to help to overcome water pollution problems.

\section{Experimental}

\subsection{Material synthesis}

CNTs, 4,5-diphenylimidazole (4,5-DPM), tetrahydrofuran (THF) and sodium hydride $(\mathrm{NaH})$ were purchased from local providers, and the alloy composition is presented in Table 1. In the functionalization of CNTs with imidazole, a mass of $30 \mathrm{mg}$ of CNT material was placed in $100 \mathrm{~mL}$ of THF contained in the round-bottom (RB) flask and continuously sonicated for $3 \mathrm{~h}$, to which $5 \mathrm{mg}$ of $\mathrm{NaH}$ were then added. Then, to this mixture, $30 \mathrm{mg}$ of 4,5-diphenyl imidazole was added, and sonication was continued for another $3 \mathrm{~h}$. The resulting mixture was refluxed for $24 \mathrm{~h}$ at $60{ }^{\circ} \mathrm{C}$. The final product was subjected to centrifugation at $6000 \mathrm{rpm}$. The black precipitate formed was washed with ethanol and deionised water. Finally, the materials were dried at $80{ }^{\circ} \mathrm{C}$ for $24 \mathrm{~h}$ in vacuum.

\subsection{Characterizations of materials}

The functionalized CNT materials were characterized using various techniques to confirm the microstructure and crystalline structure of the imidazole derivative functional groups. The chemical functional groups were studied by using a Fourier transform infrared (FTIR) spectrometer (model 65). The crystalline structure of the composite materials was studied using a Bruker $68 \mathrm{X}$-ray diffractometer (XRD), and the microstructures were studied by using a MERLIN field emission scanning

Table 1 Nickel alloy composition

\begin{tabular}{llllll}
\hline Element & $\mathrm{C}$ & $\mathrm{Cr}$ & $\mathrm{Ni}$ & $\mathrm{Nb}$ & $\mathrm{Fe}$ \\
$\%$ & 2.97 & 19.81 & 52.36 & 4.26 & 19.23
\end{tabular}

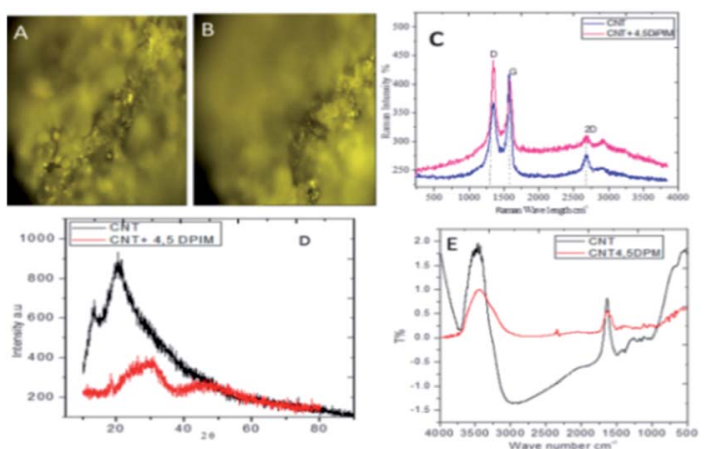

Fig. 1 Spectroscopy and XRD studies of 4,5-diphenyl-imidazolefunctionalized CNTs. (A-C) Raman spectroscopy results, (D) XRD results and (E) FTIR spectra.

electron microscope (FE-SEM) from Carl Zeiss and operated at $3.1 \mathrm{kV}$, and using a Techanic $500 \mathrm{G} 2$ transmission electron microscope (TEM) at $200 \mathrm{kV}$. The carbon disorder was analysed by using a Wintech confocal Raman spectrometer at a laser wavelength of $450 \mathrm{~nm}$.

\subsection{Corrosion inhibition studies}

Nickel alloy was purchased from a local market, cleaned, cut into $1 \mathrm{~cm} \times 1 \mathrm{~cm}$ pieces and polished with different silicon papers for improving the active surface. The polished alloys were cleaned ultrasonically for $3 \mathrm{~h}$. The alloys were mounted with cold cure epoxy materials on one side. The prepared alloys were coated with a brush with dimensions of $0.3 \mu$. The CNT composite materials were used with $3.2 \%$ polyamine as a binder. After coating, the alloys were dried for $24 \mathrm{~h}$ before using them as a working electrode. The corrosion inhibition was analysed by using a three-electrode system in an electrochemical analyzer (model CHI920D). The coated alloys were immersed for 5 days in $1 \mathrm{M} \mathrm{H}_{2} \mathrm{SO}_{4}$ to monitor the life span of the coating in a corrosive medium. The coated alloys were used as a working electrode, platinum mesh was used as a counter electrode, and $\mathrm{Ag} / \mathrm{AgCl}$ was used as a reference electrode for the electrochemical reaction. Potentiodynamic polarization was studied at a scan rate of $10 \mathrm{mV} \mathrm{s}^{-1}$. The applied potential from open circuit potential calculations was \pm 250 V. Furthermore, an impedance spectroscopy investigation was performed in order to determine the level of passivation in $1 \mathrm{M} \mathrm{H}_{2} \mathrm{SO}_{4}$ provided by coating with CNT functional materials. The applied frequency ranged from $10 \mathrm{mHz}$ to $100 \mathrm{kHz}$ to monitor the frequency dependence of passivation. The corrosion inhibition efficiency was calculated by using the equations

$$
I \%=\frac{I_{0}-I_{\mathrm{C}}}{I_{0}}
$$

and

$$
C_{\mathrm{dl}}=\frac{1}{2 \pi f R_{\mathrm{ct}}} .
$$

Here $I_{0}$ and $I_{\mathrm{C}}$ refer to the epoxy and CNT + DPM-coated nickel alloy, and $C_{\mathrm{dl}}$ refers to the double layer capacitor. 

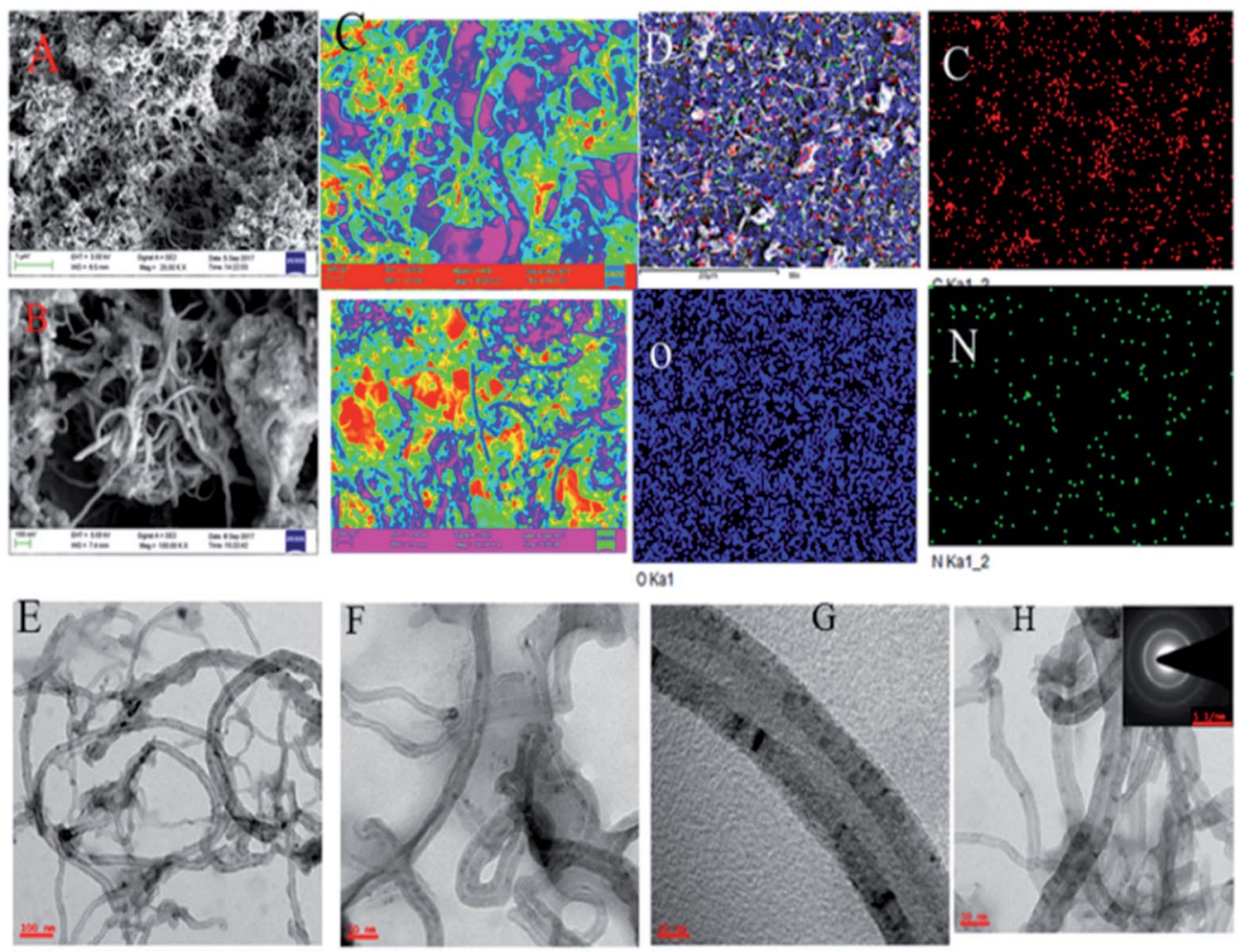

Fig. 2 (A-C) FESEM images of the microstructure of 4,5-DPM. (D) Elemental mapping images of 4,5-DPM. (E-H) TEM images of the functionalized carbon materials.

\subsection{Computational methods}

We performed theoretical studies of diphenylimidazolefunctionalized CNT using the Gaussian 09 package, revision B.01. ${ }^{18}$ All calculations were done using the hybrid DFT functional B3LYP ${ }^{19}$ and split-valence polarized basis set 6$31 \mathrm{G}^{*}{ }^{20}$ As a CNT model, we used the short $\mathrm{H}$-terminated CNT $\mathrm{C}_{100} \mathrm{H}_{20}$ and one of the edge $\mathrm{H}$-atoms was replaced with a diphenylimidazole moiety, neutral for one model and protonated for another model. We optimized the structures, calculated frequencies, and performed molecular orbital (MO) analysis of both neutral and protonated models with implicit effects from water taken into account (dielectric

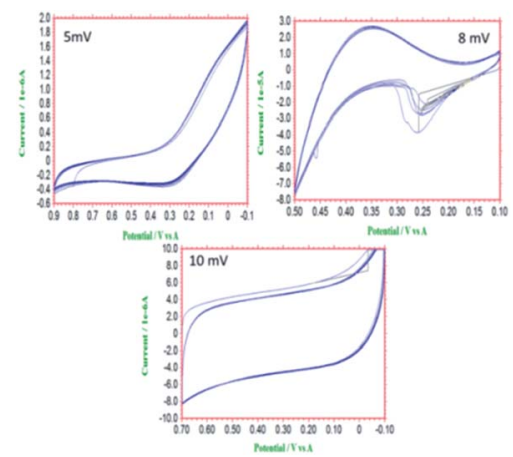

Fig. 3 Results of electrochemical stability studies of 4,5-DPM-functionalized CNT materials.
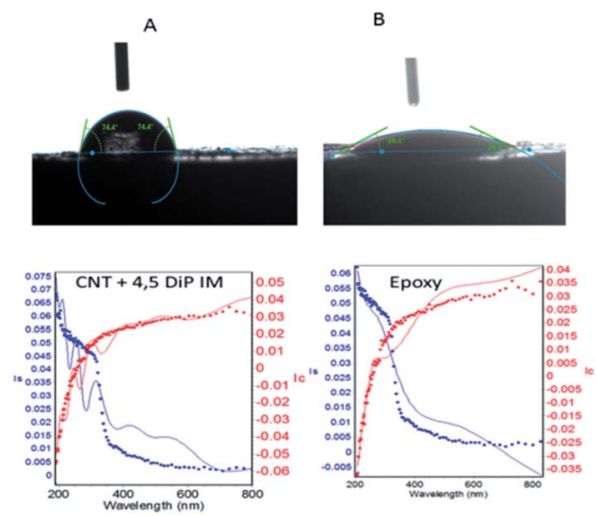

Fig. 4 Surface wettabilities of CNT + 4,5-DPM (A)- and epoxy (B)coated nickel alloys.

constant $\varepsilon=78.3553)$. With implicit solvent effects, all calculations were done using the self-consistent reaction field IEF-PCM method ${ }^{21}$ (the UFF default model used in the

Table 2 The coating thicknesses of epoxy and 4,5-DPM-functionalized CNT-coated nickel alloys

\begin{tabular}{lll}
\hline S. no. & $X^{2}$ & $\AA$ \\
\hline Epoxy & 0.29356 & 1026.733 \\
CNT + 4,5-DPM & 0.267016 & 1030.506
\end{tabular}



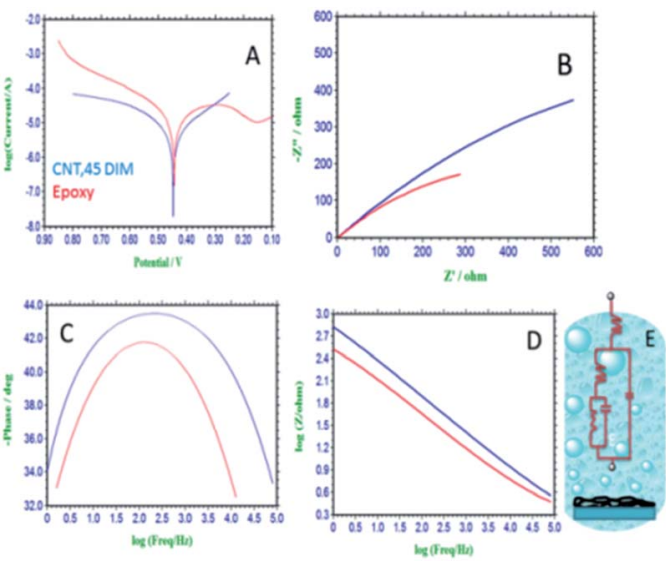

Fig. 5 Electrochemical studies of epoxy and 4,5-DPM functionalized CNT coated Mg alloy (A) Tafel curve, (B) impedance Nyquist, (C) impedance phase, (D) impedance frequency, (E) Randles circuit.

Table 3 Results of corrosion inhibition studies of 4,5-DPM + CNTand epoxy-coated nickel alloys

\begin{tabular}{lllll}
\hline S. no. & $-E_{\text {corr }} / \mathrm{mV}$ & $-I_{\text {Corr }} / \mathrm{mA}$ & $R_{\mathrm{ct}} / \mu \Omega$ & $C_{\mathrm{dl}} / \mu \mathrm{F}$ \\
\hline Epoxy & 410 & 6.255 & 980 & 6.7281 \\
CNT + 4,5-DPM & 490 & 3.505 & 990 & 3.1826 \\
\hline
\end{tabular}

Gaussian 09 package, with the electrostatic scaling factor $\alpha$ (ref. 22 ) set to 1.0 ). For the global reactivity analysis, the electrophilicity $\chi$, global hardness $\eta$, global softness $\sigma$, and nucleophilicity $\omega$ values were calculated according to the equations

$$
\begin{gathered}
\eta=\frac{I+A}{2}, \\
\chi=\frac{I-A}{2}, \\
\sigma=\frac{1}{2 \eta},
\end{gathered}
$$

and

$$
\omega=\frac{\chi^{2}}{2 \eta}
$$

\section{Results and discussion}

\subsection{Spectroscopy and XRD studies}

The spectroscopic studies of functional groups of the carbon nanotube chemically modified by imidazole are presented in Fig. 1a and b, which specifically show Raman mappings of the CNT material before and after functionalization. As shown in Fig. 1a, a very bright CNT mapping was observed, and was attributed to interactions between the carbon $\mathrm{sp}^{2}$ atoms and the laser emission. As shown in Fig. 1b, the image showed scattered features, attributed to the interactions of imidazole functional groups with CNT materials through covalent bonds. Fig. 1c shows Raman spectra of the functionalized materials and CNT.
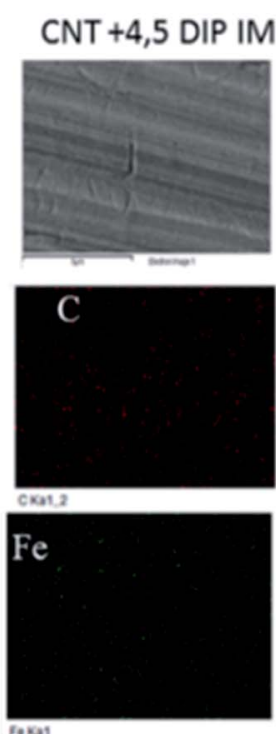

$\mathrm{Ni}$

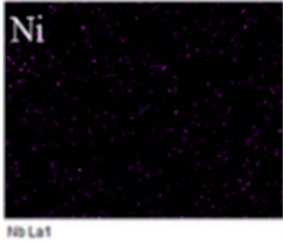

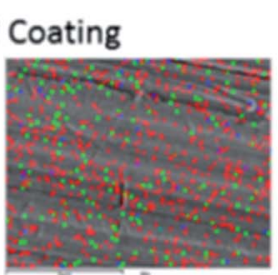
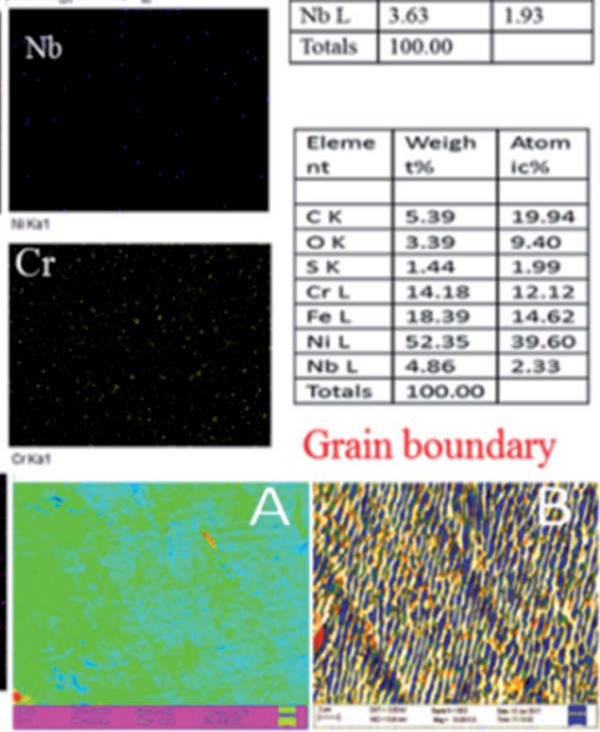

\begin{tabular}{|l|l|l|}
\hline $\begin{array}{l}\text { Eleme } \\
\mathrm{nt}\end{array}$ & Weight\% & $\begin{array}{l}\text { Atomic } \\
\%\end{array}$ \\
\hline $\mathrm{C} \mathrm{K}$ & 4.38 & 17.99 \\
\hline $\mathrm{Cr} \mathrm{L}$ & 16.68 & 15.85 \\
\hline Fe L & 20.03 & 17.72 \\
\hline Ni L & 55.28 & 46.51 \\
\hline Nb L & 3.63 & 1.93 \\
\hline Totals & 100.00 & \\
\hline
\end{tabular}

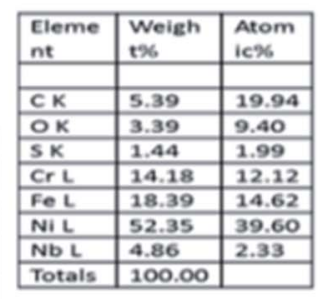

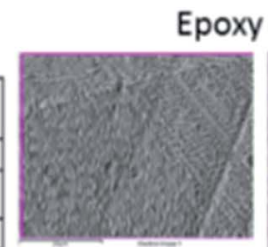

y coating
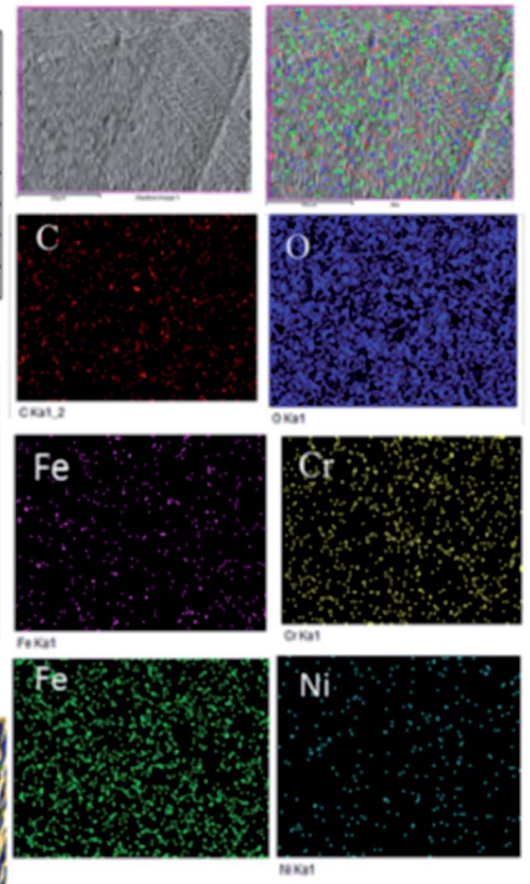

Fig. 6 The surface morphologies of epoxy-coated and 4,5-DPM + CNT-coated nickel alloys after 5 days of immersion in a corrosive medium. 


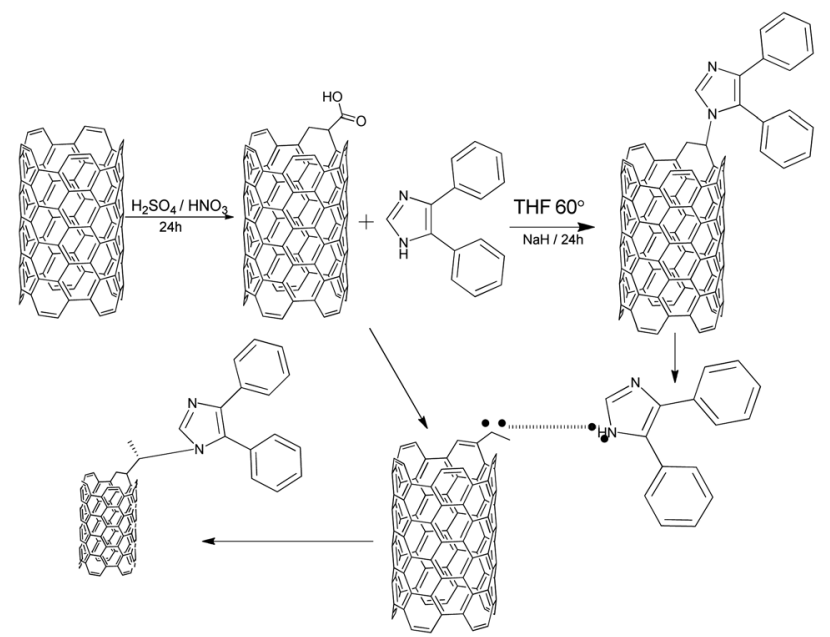

Fig. 7 A possible mechanism for the 4,5-DPM functionalization of CNTs.

The D peak at 1200 and $\mathrm{G}$ peak at $1580 \mathrm{~cm}^{-1}$ of the functionalized materials were reduced in intensity ${ }^{23}$ due to the interactions of the functionalized groups with $\mathrm{sp}^{2}$-carbon atoms., The $I_{\mathrm{D}} / I_{\mathrm{G}}$ ratio was measured to be 1.1290 for the pristine CNT, and 1.0588 for the 4,5-DPM-functionalized CNT, with this lower value suggesting secondary functionalization on the CNT side wall. Fig. 1d shows XRD results, and hence a measure of the crystallinity, of the functionalized material and CNT. The peak appearing at a 2 -theta of $10^{\circ}$ suggested that the CNT sample was polycrystalline and that the edge carbon matrix behaved as an amorphous material due to functional groups occupying the $\mathrm{sp}^{2}$ matrix. $^{24}$ Furthermore, the functionalized CNT materials showed a special broad peak appearing at a 2-theta of $45^{\circ}$, attributed to the imidazole groups covalently bound with carbon matrix. In the FTIR spectrum (Fig. 1e), a band appeared at $1500 \mathrm{~cm}^{-1}$, and was attributed to aromatic CNT matrix carbon stretching. A stretching frequency band also appeared at
$1490 \mathrm{~cm}^{-1}$, and was associated with $\mathrm{C}=\mathrm{C}$ vibration. ${ }^{25}$ And a stretching band also appeared at $2500 \mathrm{~cm}^{-1}$, and was associated with the five-membered-ring carbon atoms.

\subsection{Microstructure studies}

Fig. 2a and $\mathrm{b}$ show FESEM microstructures of 4,5-DPMfunctionalized CNT. Inspection of the image shown in Fig. 2a suggested that 4,5-DPM formed $\pi-\pi$ interactions with the carbon nanotube through covalent bonding, with free electrons of the imidazole nitrogen electrostatically interacting with the nanotube matrix. ${ }^{26}$ The examined elements were regularly distributed over the CNT matrix. Fig. 2c shows dark green colour for nitrogen interactions with CNT carboxylic groups and nitrogen $\pi-\pi$ electrons interacting with the carbon matrix. Fig. 2e shows the acquired TEM image of the 4,5-DPMfunctionalized nanotube; the dark carbon dot observed in the middle of the CNT was due to the presence of imidazole. This conclusion was confirmed by inspecting the images shown in Fig. $2 \mathrm{f}$ and $\mathrm{g}$, with Fig. $2 \mathrm{~g}$ showing the open wall CNT.

\subsection{Electrochemical stability}

The functionalized materials were studied for their electrochemical stability in the three-electrode system. We investigated the redox stabilities using different applied potentials. As seen in Fig. 3a, at $5 \mathrm{mV} \mathrm{s}^{-1}$, five cycles did not change the $\mathrm{CV}$ pattern due to the structural stability at low voltage. With an increase of applied voltage to $8 \mathrm{mV}$, the $\mathrm{CV}$ pattern changed and showed a broad redox peak, due to delocalization of the electrons of the host molecule imidazole five-membered ring. ${ }^{27,28}$ Furthermore, with the increase of the applied potential to $10 \mathrm{mV}$, the stability of redox behaviour did not change, due to the electrostatic attraction between the imidazole and the carbon matrix. ${ }^{29}$ The electrochemical study showed excellent stability of the functionalized material in the studied acidic environments.
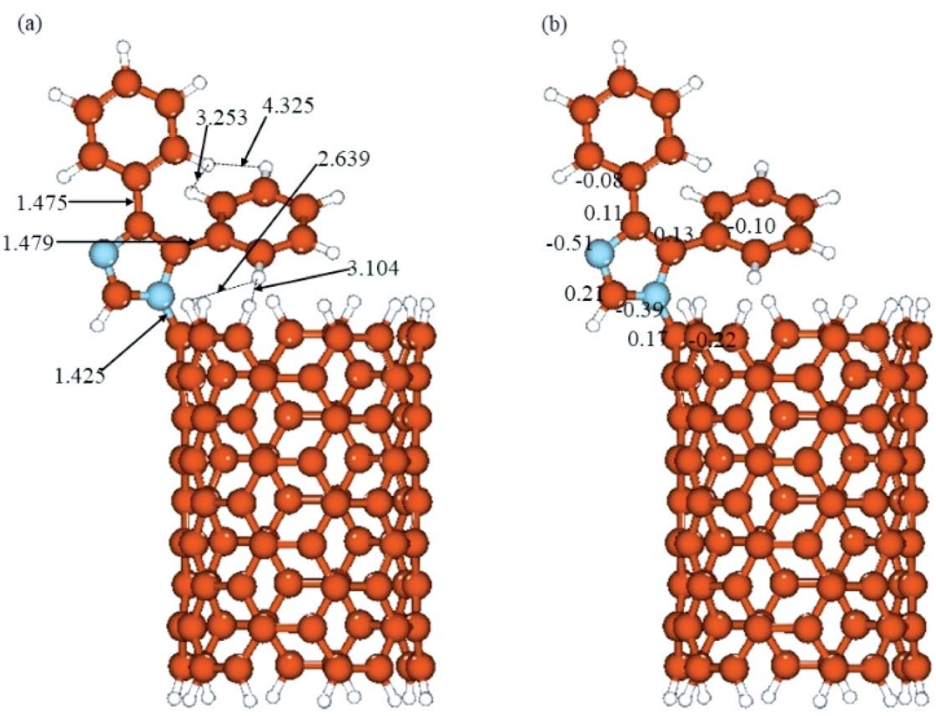

Fig. 8 (a) Structure and (b) NBO charges of the neutral CNT-diphenylimidazole model optimized with implicit effects from water. 


\subsection{Coating thickness and surface wettability}

Fig. 4 shows the results of coating thickness studies for the epoxy and 4,5-DPM functionalized CNT-coated nickel alloy. 4,5DPM-functionalized CNT showed a wettability angle of about $85^{\circ}$, and epoxy-coated nickel alloy showed a wettability angle of about around $26^{\circ} \cdot \cdot^{30-32}$ The surface wettability results were consistent with the results of spectroscopy studies. As listed in Table 2, the epoxy-coated nickel alloy showed a thickness of about $1026 \AA$, which suggested that in this epoxy-coated nickel, only epoxy functional groups were present. ${ }^{33,34}$ The 4,5-DPMfunctionalized CNT-coated nickel alloy showed an approximately $200 \mathrm{~nm}$ wave form, due to the presence of hydroxyl groups and other active functional groups present at the coating surface.

\subsection{Corrosion inhibition studies}

Fig. 5 presents the results of electrochemical stability studies of the epoxy- and 4,5-DPM-functionalized CNT-coated nickel alloys. The epoxy-coated nickel alloy surface demonstrated pitting corrosion initiated by corrosive ion attack. Corrosion current densities increased, due to the alloy having undergone corrosion. ${ }^{35-38}$ Corrosion potential decreased, due to cathodic hydrogen evolution, and indicating that anodic alloy dissolution increased. The 4,5-DPM-functionalized CNT carbon-coated nickel alloy corrosion current densities decreased and corrosion potential increased due to strong physisorption of the 4,5-DPMfunctionalized CNT on the alloy surface. Thereby, hydrogen evolution was suppressed by the 4,5-DPM-functionalized CNT materials blocking the active alloy surface. Hence, CNT functionalized materials resulted in an increase in coating passivation. The corrosion inhibition values are presented in Table 3. Fig. 5B-D show results of impedance spectroscopy of epoxycoated nickel alloy. Nyquist semicircles were observed to be suppressed, as the corrosive ions diffused to the coating due to the poor passivation provided by the epoxy coating on the alloy surface. Furthermore, the solution resistance and charge transfer values were decreased, due to hydrogen evolution on the alloy surface. Therefore, epoxy coating failed to provide long-duration durability in harsh environments. ${ }^{39-41}$ For the nickel alloys coated with 4,5-DPM-functionalized materials, the sizes of the Nyquist semicircles were increased, attributed to the strong adsorption of the active functionalized materials on the alloy surface. Furthermore, the impedance frequency and impedance phase angle were observed to be increased, attributed to strong chemisorption of the CNT functionalized carbon materials on the alloys. The double layer capacitance values were observed to be decreased by effect of the CNT functionalized materials. The impedance values are presented in Table 2. The impedance spectroscopy and potentiodynamic polarization studies revealed 4,5-DPM-functionalized CNT to be an excellent corrosion-retarding material in acidic medium.

\subsection{Alloy microstructure studies}

Fig. 6 shows, the surface morphologies for the epoxy- and 4,5DPM-functionalized CNT-coated nickel alloys after five days of immersion in a corrosive medium. For the epoxy-coated nickel alloy, uniform corrosion occurred, attributed to the epoxy coating peeling off from the alloy surface. However, the physisorption on the alloy surface was also weak, due to the initiation of pitting corrosion on this epoxy-coated nickel alloy surface. And the epoxy-coated nickel alloy grain boundaries were severely affected due to the poor epoxy coating stability, as shown in Fig. 6b. Furthermore, the lack of protective groups on the epoxy coating resulted in this epoxy not having sufficient protection on the coating. However, the 4,5-DPM-functionalized CNT-coated nickel alloy showed a smooth surface and localized corrosion diminished by the CNT functionalized coating materials. The CNT non-bonding electrons were implicated in physisorption of the material on the nickel alloy surface and imidazole nitrogen free electrons were implicated in chemisorption on the nickel alloys. ${ }^{4-46}$ Thus, an excellent corrosion (a)

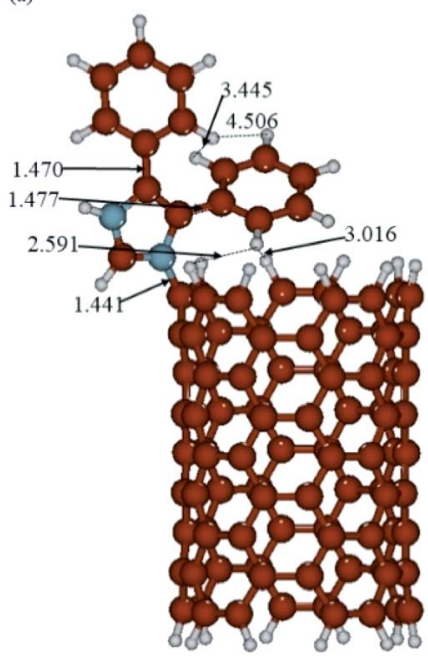

(b)

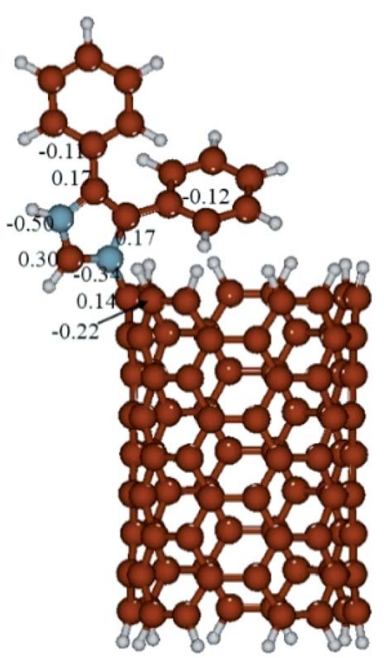

Fig. 9 (a) Structure and (b) NBO charges of the protonated CNT-diphenylimidazole model optimized with implicit effects from water. 
barrier coating layer due to the strong physisorption on the alloy surface was created. The coating of functionalized materials also reduced the occurrence of hydrogen evolution. However, no oxygen element was detected on the surface, because the alloy did not become corroded by any aggressive oxygen-containing species. The corrosive ions were not able to penetrate the coated layer because of strong hydrophobic nature of CNT as shown from the surface wettability results.

\subsection{Mechanism of 4,5-DPM functionalization of the CNT}

Fig. 7 shows a proposed mechanism of the 4,5-DPM functionalization of the CNT. According to this proposal, subjecting the CNTs to acidic conditions resulted in opening up the functionalized side, after which a cyclo addition on the CNT took place, with the imidazole primary nitrogen atom sharing a pair of electrons with the CNT carboxylic cation to form the covalent $\mathrm{N}-\mathrm{C}$ bond. ${ }^{47-49}$

\subsection{Computational studies}

The optimized structures and calculated NBO charges of the neutral and protonated CNT-diphenylimidazole models calculated with the implicit water effects are provided in Fig. 8 and 9, respectively, and two unoccupied and six occupied molecular orbitals of these models are presented in Fig. 10 and 11, respectively. In our calculations, as can be seen from Fig. 8a and 9a, the CNT structure did not become distorted upon binding the diphenylimidazole molecule, both for neutral and protonated forms, and the phenyl group of diphenylimidazole, located next to the end of the CNT, avoided close contacts with the CNT for both the neutral and protonated structures as indicated by the $c a$. 2.59-3.11 A distances between this moiety and the CNT. ${ }^{50}$
Comparison of the neutral and protonated species showed quite similar structures. Relative to the neutral structure, in the protonated structure, the $\mathrm{C}-\mathrm{C}$ bonds between the imidazole and two phenyl moieties were just 0.002-0.005 $\AA$ shorter, and the $\mathrm{C}(\mathrm{CNT})-\mathrm{N}($ imidazole $)$ bond $0.016 \AA$ longer, with distances between the horizontally aligned phenyl moiety and the CNT shortened by $c a$. 0.05-0.09 A. Also, the distances between the horizontally and vertically aligned phenyl moieties were $c a$. 0.18-0.19 A longer.

During the binding of the diphenylimidazole unit (neutral or protonated) to the CNT, a significant transfer of charge was calculated to occur to the $\mathrm{N}$-center from the $\mathrm{C}$-atom connected to the diphenylimidazole, ca. 0.36-0.38e (compared to the charges at the $\mathrm{C}$-atom connected to the diphenylimidazole, $0.17 / 0.14 e$, and at the neighbouring C-atoms of the CNT, $-0.21 /$ $-022 e$ ) (Fig. 8b and 9b). Significant negative charge, $c a$. $-0.50 e$, was also calculated to accumulate on another $\mathrm{N}$-atom of the diphenylimidazole ( $c f$. Fig. $8 \mathrm{~b}$ and $9 \mathrm{~b}$ ). In the protonated species, the positive charge was calculated to be partially smeared over the imidazole moiety, which could be seen from the slightly higher positive charge on its $\mathrm{C}(\mathrm{H})$-atom and slightly decreased negative charge on its $\mathrm{N}-\mathrm{C}(\mathrm{CNT})$ atom.

Analysis of the molecular orbitals of the neutral and protonated CNT-diphenylimidazole models showed several features (Fig. 10 and 11). (i) The HOMO and LUMO/LUMO+1 of the neutral model were calculated to be dominated by contributions from the CNT, whereas HOMO-1 - HOMO-3 and HOMO-5 were calculated to have significant contributions from the diphenylimidazole moiety as well. (ii) The HOMO and $\mathrm{LUMO} / \mathrm{LUMO}+1$ of the protonated model were also determined to be dominated by contributions from the CNT, a feature also
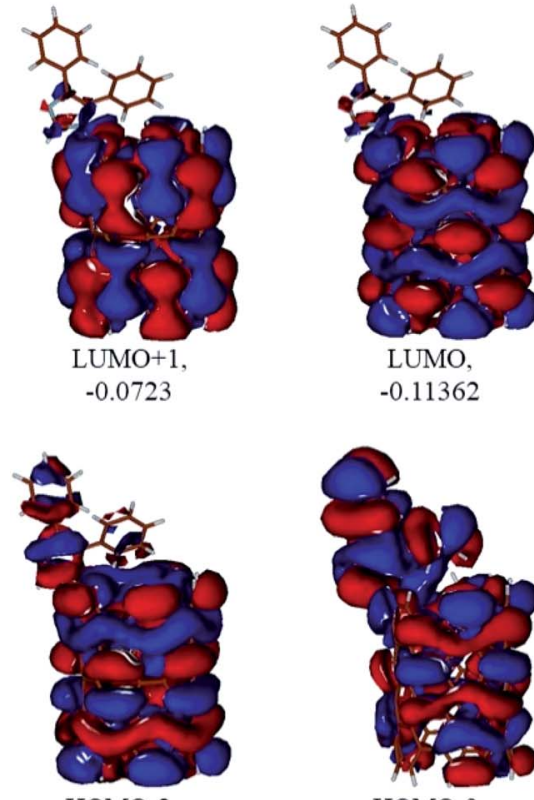

HOMO-2, $-0.20075$

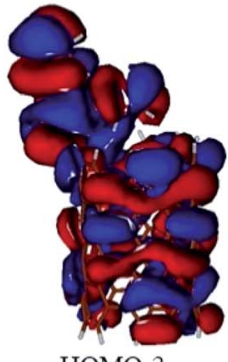

HOMO-3, $-0.21038$
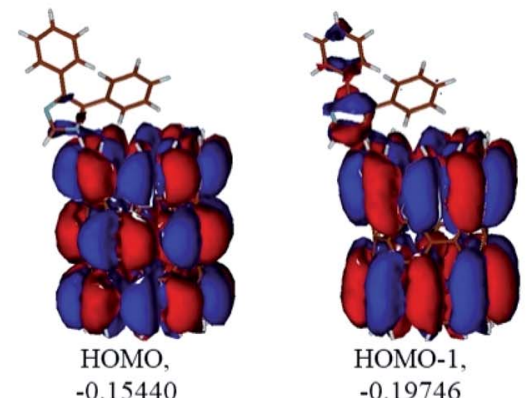

$-0.19746$

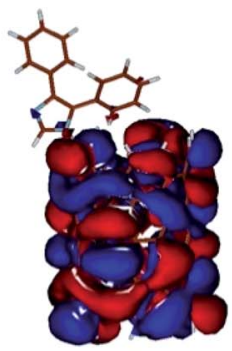

HOMO-4, $-0.21433$

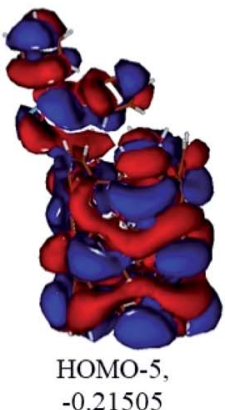

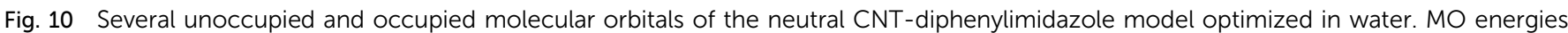
given in $A . U$ 


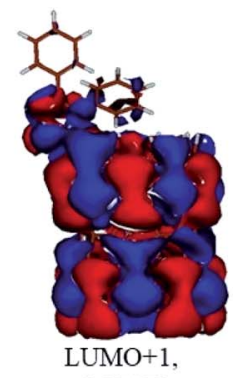

$-0.07573$

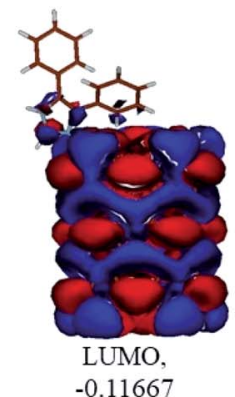

$-0.11667$
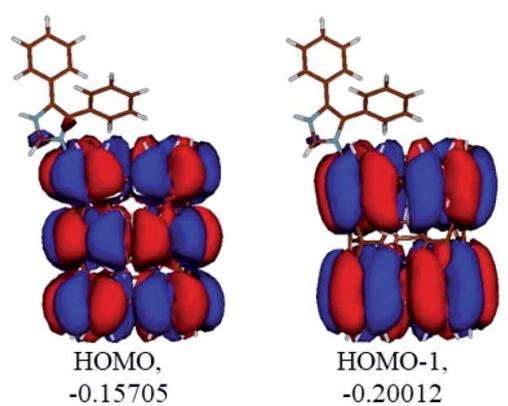

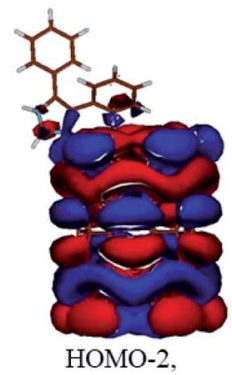

$-0.20371$

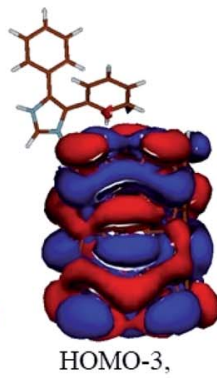

$-0.21638$

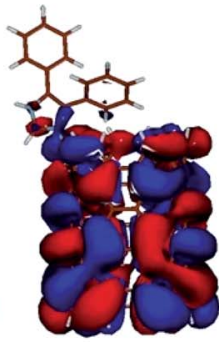

HOMO-4,

$-0.21714$

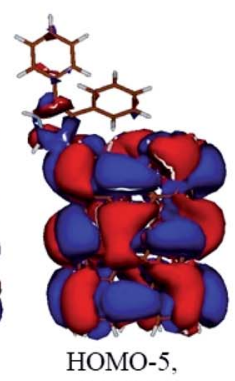

$-0.22000$

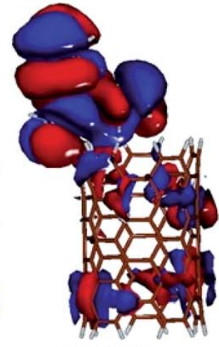

HOMO-8,

$-0.24426$

Fig. 11 Several unoccupied and occupied molecular orbitals of the protonated CNT-diphenylimidazole model optimized in water. MO energies given in A.U.

indicated for HOMO-1 - HOMO-7 (HOMO-6 and HOMO-7 are not shown in Fig. 11), whereas HOMO-8 of this model was determined to be strongly dominated by contributions from the diphenylimidazole moiety, a feature differing between the protonated model and the neutral model. (iii) The energy difference between HOMO and HOMO-1/HOMO-2 for each of the two models was found to be not too significant, $c a$. 1.2$1.3 \mathrm{eV}$. Thus, participation of these orbitals in (electro) chemical processes should be considered possible. (iv) In the protonated model, all of the MOs were calculated to be somewhat more stable, and hence predicted to be slightly less reactive, than for the neutral model. (v) Our calculations indicated the global hardness $\eta$ values of the both CNT-diphenylimidazole models to be relatively low and the global softness $\sigma$ values to be quite noticeable, specifically $c a$. 27-28 times greater than the global hardness values, as summarized in Table 4 (the global reactivity parameters were computed using energies of two couples of MOs of the system studied, HOMO \& LUMO and HOMO-1 \& LUMO-1). ${ }^{51-57}$ This result suggested that this system would be highly reactive towards oxidizing agents attacking the surface. This conclusion was also supported by relatively small HOMOLUMO energy gap value $(\Delta E)$ for the CNT-diphenyl imidazole models (Table 4). Low global electronegativity $(\chi)$ and electrophilicity $(\omega)$ values suggested high potential reactivity of the CNT-diphenylimidazole system with oxidizing electrophilic agents.

The combination of the calculated parameters - relatively narrow HOMO/LUMO gap along with the MO composition and computed global reactivity parameters and NBO charges suggested that the CNT-diphenylimidazole material would interact well with various oxidizers, thus protecting the Ni alloy surface and acting as a corrosion inhibitor. Interestingly, for both the neutral and protonated CNT-diphenylimidazole models, very similar calculated global reactivity parameters values were obtained, indicating that both models would have similar reactivities towards oxidizing agents and thus quite similar anticorrosion properties.

Table 4 Global reactivity parameters computed for the neutral and protonated CNT-diphenylimidazole models with implicit effects from water. Values in A.U. A = HOMO and LUMO, B= HOMO-1 and LUMO-1

\begin{tabular}{|c|c|c|c|c|c|c|c|c|}
\hline & $E_{(\text {HOMO/LUMO) }}$ & $\Delta E$ & $I$ & $A$ & $\chi$ & $\sigma$ & $\eta$ & $\omega$ \\
\hline \multicolumn{9}{|c|}{ Neutral model } \\
\hline A & $-0.15440 /-0.11362$ & 0.0408 & 0.15440 & 0.11362 & 0.02039 & 3.73106 & 0.13401 & 0.00155 \\
\hline \multicolumn{9}{|c|}{ Protonated model } \\
\hline $\mathrm{A}$ & $-0.15705 /-0.11667$ & 0.0404 & 0.15705 & 0.11667 & 0.02019 & 3.65337 & 0.13686 & 0.001489 \\
\hline
\end{tabular}




\subsection{Corrosion mechanisms}

The 4,5-diphenyl-imidazole-functionalized CNT could support electron redistribution to the nickel alloy surface. Hence, the free electrons of the primary nitrogen of the 5-membered imidazole ring would delocalize to the nearby positively charged nickel alloy surface, and thus the CNT would act as a booster for electron delocalization due to the strongly electron-donating multifunctional ring and non-bonding electrons contributing to the transfer of electrons to the electron-deficient alloy surface. The surface wettability studies were supported by electrochemical results. The CNT could be physisorbed on the alloy surface. Furthermore, the DFT results suggested that electrons could be donated to the alloy surface. And the hardness and softness values supported the electron donation to the nickel alloy surface. Finally, the imidazole derivative was indicated to act as an excellent donor of electrons to the nickel alloy surface.

\section{Conclusions}

4,5-Diphenyl-imidazole-functionalized CNTs have been studied as a corrosion inhibitor. The spectroscopy studies revealed that the imidazole derivative covalently functionalized the CNT matrix. Furthermore, XRD results confirmed its crystallinity. The microscopy studies suggested that the CNT formed a continuous tubular structure a few nanometres long. The corrosion inhibition studies, including the DFT studies, suggested that the functionalized materials could provide excellent inhibition of corrosion of the nickel alloy surface. We expect the imidazole-derivative-functionalized CNTs to find use as a green corrosion inhibitor in the future.

\section{Conflicts of interest}

There are no conflicts to declare.

\section{Acknowledgements}

N. P. is thankful for a Non-NET fellowship and to the central instruments facilities of the Central University of Gujarat. The author would like to thank Prof M. V. Rajasekharan (School of Chemical Sciences, University of Hyderabad) for extending his laboratory facilities under the UGC-NRC scheme.

\section{References}

1 M. Qiao and C. Zhou, Corros. Sci., 2021, 63, 239-245.

2 Y. X. Xu, J. T. Lu, W. Y. Li, Z. Yang, Y. Y. Dang and X. W. Yang, Corros. Sci., 2018, 143, 148-156.

3 S. Anwar, Y. Zhang and F. Khan, RSC Adv., 2018, 8, 2886128873.

4 J. J. Goveas, S. Shetty, N. P. Mascarenhas, A. C. Hegde and R. A. Gonsalves, New J. Chem., 2018, 42, 13660-13666.

5 Z. Feng, L. Ren, J. Zhang, P. Yang and M. An, RSC Adv., 2016, 6, 42029-42040.
6 K. Miyauchia, H. Watanabe and M. YuasacaIchikawa, Prog. Org. Coat., 2016, 94, 73-78.

7 B. Gregoirea, X. Montero, M. C. Galetz, G. Bonneta and F. Pedraza, Corros. Sci., 2018, 141, 211-220.

8 S. H. Cho, S. C. Kwon, D. Y. Kim and J. H. Lee, Corros. Sci., 2019, 151, 20-26.

9 T. Chen, H. John, J. Xu, Q. Lu, J. Hawk and X. Liu, Corros. Sci., 2014, 78, 151-161.

10 L. Zhang, L. Wang, C. M. B. Holt, B. Zahiri, Z. Li, K. Malek, T. Navessin, M. H. Eikerling and D. Mitlin, Energy Environ. Sci., 2012, 5, 6156-6172.

11 A. M. Kumar, M. M. Rahman and Z. M. Gasem, RSC Adv., 2015, 5, 63537-63544.

12 H. R. B. Rad, M. Abdellahi, E. Hamzah, M. Daroonparvar and M. Rafiei, $R S C A d v$., 2016, 6, 108498-108512.

13 W. A. D. M. Jayathilaka, A. Chinnappan and S. Ramakrishna, J. Mater. Chem. C, 2017, 5, 9209-9237.

14 J. H. Huh, S. H. Kim, J. H. Chu, S. Y. Kim, J. H. Kim and S. Y. Kwon, Nanoscale, 2014, 6, 4379-4386.

15 Y. Liu, J. Zhang, S. Li, Y. Wang, Z. Hana and L. Ren, RSC Adv., 2014, 4, 45389-45396.

16 S. Dea and J. L. Lutkenhaus, Green Chem., 2018, 20, 506-514. 17 (a) N. N. Aung, W. Zhou, C. S. Goh, S. M. L. Nai and J. Wei, Corros. Sci., 2010, 52, 1551-1553; (b) Q. Do, H. An, G. Wang, G. Meng, Y. Wang, B. Liu, J. Wang and F. Wang, Corros. Sci., 2019, 147, 246-259.

18 M. J. Frisch, G. W. Trucks, H. B. Schlegel, G. E. Scuseria, M. A. Robb, J. R. Cheeseman, G. Scalmani, V. Barone, G. A. Petersson, H. Nakatsuji, X. Li, M. Caricato, A. Marenich, J. Bloino, B. G. Janesko, R. Gomperts, B. Mennucci, H. P. Hratchian, J. V. Ortiz, A. F. Izmaylov, J. L. Sonnenberg, D. Williams-Young, F. Ding, F. Lipparini, F. Egidi, J. Goings, B. Peng, A. Petrone, T. Henderson, D. Ranasinghe, V. G. Zakrzewski, J. Gao, N. Rega, G. Zheng, W. Liang, M. Hada, M. Ehara, K. Toyota, R. Fukuda, J. Hasegawa, M. Ishida, T. Nakajima, Y. Honda, O. Kitao, H. Nakai, T. Vreven, K. Throssell, J. A. Montgomery Jr, J. E. Peralta, F. Ogliaro, M. Bearpark, J. J. Heyd, E. Brothers, K. N. Kudin, V. N. Staroverov, T. Keith, R. Kobayashi, J. Normand, K. Raghavachari, A. Rendell, J. C. Burant, S. S. Iyengar, J. Tomasi, M. Cossi, J. M. Millam, M. Klene, C. Adamo, R. Cammi, J. W. Ochterski, R. L. Martin, K. Morokuma, O. Farkas, J. B. Foresman and D. J. Fox, Gaussian 09, Revision B.01, Gaussian, Inc., Wallingford CT, 2016.

19 R. Parr and W. G. Yang, Density-functional theory of atoms and molecules, Oxford University Press, Oxford, 1989.

20 A. McLean and D. G. S. Chandler, J. Chem. Phys., 1980, 72, 5639-5648.

21 E. Cancès and B. T. Mennucci, J. Chem. Phys., 1997, 107, 3032-3041.

22 V. Barone, M. Cossi and J. Tomasi, J. Chem. Phys., 1997, 107, 3210-3221.

23 S. X. Lima, K. W. Woob, J. Ng, J. Lua, S. Y. Kwanga, Z. Zhang, E. S. Toka and C. H. Sowa, Nanoscale, 2015, 7, 17538-17546.

24 Y. Xu, H. Xu, L. Wang, J. Yan, H. Li, Y. Song, L. Huang and G. Cai, Dalton Trans., 2013, 42, 7604-7613. 
25 X. Cao, S. Jia, W. Huang, Y. Tang, J. O. Duus, J. Lou and Q. Chi, J. Mater. Chem. A, 2019, 7, 7501-7515.

26 A. Majumder, M. Khazaee, J. Opitz, E. Beyer, L. Baraban and G. Cuniberti, Phys. Chem. Chem. Phys., 2013, 15, 1715817164.

27 R. Giovanardi, M. Montorsi, G. Ori, J. Cho, T. Subhani, A. R. Boccaccini and C. Siligardi, J. Mater. Chem., 2010, 20, 308-313.

28 Z. Feng, L. Ren, J. Zhang, P. Yang and M. An, RSC Adv., 2016, 6, 88469-88485.

29 R. Konnol and K. Joseph, RSC Adv., 2016, 6, 23887-23899.

30 M. Musameh, C. P. Huynh, M. Hickey and I. L. Kyratzis, Analyst, 2016, 141, 2748-2755.

31 H. W. Chang, Y. R. Lu, J. L. Chen, C. L. Chen, J. F. Lee, J. M. Chen, Y. C. Tsai, C. M. Chang, P. H. Yeh, W. C. Chou, Y. H. Lioug and C. L. Dong, Nanoscale, 2015, 7, 1725-1735.

32 L. He, A. Karumuri and S. M. Mukhopadhy, RSC Adv., 2017, 7, 25265-25275.

33 Y. Yang, C. Nie, Y. Deng, C. Cheng, C. He, L. Ma and C. Zhao, RSC Adv., 2016, 6, 88265-88276.

34 S. Zhou, J. Sheng, Z. Yang and X. Zhang, J. Mater. Chem. A, 2018, 6, 8763-8771.

35 J. Sun, Q. Zhang, R. Ding, H. Lv, H. W. Yan, X. Yuan and Y. Xu, Phys. Chem. Chem. Phys., 2014, 16, 16684-16693.

36 R. E. Roy, B. Soundiraraju, D. Thomas, N. Balachandran, C. Ambika and R. S. Rajeev, New J. Chem., 2017, 41, 1215912171.

37 C. C. Ling, Q. Z. Xue, N. N. Jinga and D. Xia, RSC Adv., 2012, 2, 7549-7556.

38 E. N. Zare, M. M. Lakouraj, S. Ghasemi and E. Moosavia, RSC $A d v .$, 2015, 5, 68788-68795.

39 Y. H. Wen, L. Shao, P. C. Zhao, B. Y. Wang, G. P. Cao and Y. S. Yang, J. Mater. Chem. A, 2017, 5, 15752-15758.

40 S. K. Krishnan, E. Singh, P. Singh, M. Meyyappan and H. S. Nalwa, RSC Adv., 2019, 9, 8778-8881.
41 T. Liu, L. Zhao, D. Wang, J. Zhu, B. Wang and C. Guo, RSC Adv., 2013, 3, 25648-25651.

42 Z. Feng, M. An, L. Ren, J. Zhang, P. Yanga and Z. Chen, RSC Adv., 2016, 6, 64726-64740.

43 J. J. Goveas, S. Shetty, N. P. Mascarenhas, A. C. Hegde and R. A. Gonsalves, New J. Chem., 2018, 42, 13660-13666.

44 S. Anwar, Y. Zhang and F. Khan, RSC Adv., 2018, 8, 2886128873.

45 Z. Mohammadpour and H. R. Zare, New J. Chem., 2018, 42, 5425-5432.

46 S. Khameneh-asl, A. Farzaneh, H. Teymourinia, O. Mermerc and M. G. Hosseini, RSC Adv., 2016, 6, 78774-78783.

47 Z. Feng, L. Ren, J. Zhang, P. Yang and M. An, RSC Adv., 2016, 6, 42029-42040.

48 K. Miyauchi, H. Watanabe and M. YuasacaIchikawa, Prog. Org. Coat., 2016, 94, 73-78.

49 F. Yang, H. Kang, E. Guo, R. Li, Z. Chen, Y. Zeng and T. Wang, Corros. Sci., 2018, 139, 333-345.

50 S. Hua, L. Liua, Y. Cuia, Y. Lia and F. Wanga, Corros. Sci., 2019, 146, 202-212.

51 O. Zabihi, M. Ahmadi, M. A. bagherjeri and M. Naebe, $R S C$ Adv., 2015, 5, 98692-98699.

52 R. Malik, C. McConnell, N. T. Alvarez, M. Haase, S. Gbordzoe and V. Shanov, RSC Adv., 2016, 6, 108840-108850.

53 Z. Qi, Y. Tan, Z. Zhang, L. Gao, C. Zhang and J. Tiana, RSC Adv. , 2018, 8, 38689-38700.

54 F. Moradi, M. D. Ganji and Y. Sarrafia, Phys. Chem. Chem. Phys., 2017, 19, 8388-8399.

55 E. Soleymani, H. Alinezhad, M. D. Ganji and M. Tajbakhsh, J. Mater. Chem. B, 2017, 5, 6920-6929.

56 M. Verma and P. A. Deshpande, Phys. Chem. Chem. Phys., 2017, 19, 8757-8767.

57 A. Sengupta, J. Sk, A. Boda and S. M. Ali, RSC Adv., 2016, 6, 39553-39562. 